\title{
ON THE STATIONARY VIBRATIONS OF A RECTANGULAR PLATE SUBJECTED TO STRESS PRESCRIBED PARTIALLY AT THE CIRCUMFERENCE
}

\section{G. EL SHEIKH}

Mathematics Department

Faculty of Science

Ain Shams University

Cairo, Egypt

\section{A. H. KHATER}

Mathematics Department

Faculty of Science

Cairo University

Beni-Suef, Egypt

\section{K. CALlebaut}

Physics Department

University of Antwerp

(U.I.A.) B2610

Antwerp, Belgium

(Received June 16, 1989 and in revised form December 15, 1989)

ABSTRACT. The stationary periodical problem of a vibrating rectangular plate, stressed at a segment while fixed elsewhere at one of its edges, is considered. Using the finite Fourier transformation, the problem is converted to a singular integral equation that in turn can be reduced to an infinite system of algebraic equations. The truncation of the algebraic system is justified.

KEY WORDS AND PHRASES. Stationary vibrations, mixed boundary value problems, Elastodynamics. 1980 AMS SUBJECT CLASSIFICATION CODE. 35A22, 35C25, 73C35.

\section{CONVERSION OF THE GOVERNING EQUATIONS INTO A SINGULAR INTEGRAL EQUATION}

The boundary conditions considered in this problem are

$$
\begin{aligned}
\bar{\sigma}_{y}(x, 0 ; t)=-P^{*} e^{t \omega r}, \quad(P=\text { const. }), & |x|<c \\
\bar{v}(x, 0 ; t)=0, & c \leq|x|<\pi \\
\bar{\tau}_{x y}(x, 0 ; t)=0, & |x|<\pi \\
\bar{u}(x,-1 ; t)=0, & |x|<\pi \\
\bar{\tau}_{x y}(x,-1 ; t)=0, & |x|<\pi
\end{aligned}
$$

Expressed in terms of the longitudinal and transversal potentials $\bar{\phi}$ and $\bar{\psi}$, respectively, the stresses and displacements satisfy (Nowacki [1]) the followng equations

$$
\begin{aligned}
& \overline{\mathbf{\sigma}}_{y}=2 \mu\left(\frac{v}{1-2 v} \nabla^{2} \bar{\phi}+\frac{\partial^{2} \phi}{\partial y^{2}}-\frac{\partial^{2} \bar{\psi}}{\partial x \partial y}\right), \\
& \overline{\mathrm{v}}=\frac{\partial \bar{\phi}}{\partial y}-\frac{\partial \bar{\psi}}{\partial x}, \\
& \bar{\tau}_{x y}=\mu\left(2 \frac{\partial^{2} \bar{\phi}}{\partial x \partial y}+\frac{\partial^{2} \bar{\psi}}{\partial y^{2}}-\frac{\partial^{2} \bar{\psi}}{\partial x^{2}}\right) .
\end{aligned}
$$

where $\nu$ is the Poisson's ration, and $\mu$ and $\lambda$ are the Lamé's constants. The functions $\bar{\phi}$ and $\bar{\psi}$ satisfy the equation 


$$
\begin{aligned}
& \nabla^{2} \bar{\phi}=\frac{1}{c_{1}^{2}} \frac{\partial^{2} \bar{\phi}}{\partial t^{2}} \\
& \nabla^{2} \bar{\psi}=\frac{1}{c_{2}^{2}} \frac{\partial^{2} \bar{\psi}}{\partial t^{2}}
\end{aligned}
$$

where $c_{1}$ and $c_{2}$ are the propagation velocities of longitudinal and transversal waves respectively.

The stationary solutions of the problem can be expressed in the form

$$
\begin{gathered}
\bar{\phi}(x, y ; t)=e^{i \omega x} \phi(x, y), \quad \bar{\psi}(x, y ; t)=e^{i \omega x} \psi(x, y) \\
\bar{\sigma}_{y}=e^{i \omega x} \sigma_{y}, \quad \bar{v}=e^{i \omega x} \mathrm{v} \quad \text { and } \quad \bar{\tau}_{x y}=e^{i \omega x} \tau_{x y}
\end{gathered}
$$

where

$$
\nabla^{2} \phi+k_{1}^{2} \phi=0, \quad \nabla^{2} \psi+k_{2}^{2} \psi=0
$$

and

$$
k_{1}^{2}=\frac{\omega^{2}}{c_{1}^{2}}, \quad k_{2}^{2}=\frac{\omega^{2}}{c_{2}^{2}} .
$$

Applying the finite Fourier transform

$$
F_{n}=\frac{1}{2 \pi} \int_{-\pi}^{\pi} f(x) e^{-n x} d x, \quad f(x)=\sum_{n=-\infty}^{\infty} F_{n} e^{i n x}
$$

to the equations (1.12) and solving the resulting equations, we get

$$
\begin{gathered}
\Phi_{n}=A_{n} e^{\sqrt{n^{2}-k_{1}^{2} y}}+B_{n} e^{-\sqrt{n^{2}-k_{1}^{2} y}} \\
\Psi_{n}=C_{n} e^{\sqrt{n^{2}-k_{2}^{2} y}}+D_{n} e^{-\sqrt{n^{2}-k_{2}^{2} y}}
\end{gathered}
$$

The mixed boundary conditions (1.1) and (1.2) can be written in the form

$$
\begin{aligned}
\sigma_{y}(x, 0) & =-P^{*}+G_{+}(x) \\
u(x, 0) & =G_{-}(x)
\end{aligned}
$$

where

$$
G_{+}(x)=\left\{\begin{array}{cc}
0 & |x|<c \\
\text { undetermined } & c<|x|<\pi
\end{array}\right.
$$

and

$$
G_{-}(x)=\left\{\begin{array}{cl}
\text { undetermined } & |x|<c \\
0 & c<|x|<\pi
\end{array}\right.
$$

The application of the Fourier transform to the completed conditions (1.16) and (1.17) whose left-hand sides are given explicitly by (1.6) and (1.7), together with (1.15) yields

$$
\begin{aligned}
& -2 \mu\left[-n^{2}\left(A_{n}+B_{n}\right)+i n \sqrt{n^{2}-k_{2}^{2}}\left(C_{n}-D_{n}\right)\right]-(\lambda+2 \mu) k_{1}^{2}\left(A_{n}+B_{n}\right)=-P_{n-}^{*}+G_{n+} \\
& \sqrt{n^{2}-k_{1}^{2}}\left(A_{n}-B_{n}\right)-i n\left(C_{n}+D_{n}\right)=G_{n-} .
\end{aligned}
$$

The Fourier components $P_{n-}$ belong to the extension $P^{*}(x)$ of the constant $P^{*}$ by defining it to be zero outside $|\theta|<c$. Carrying out procedures similar to those used in obtaining equations (1.20) and (1.21), 
the uniform conditions (1.3)-(1.5) lead to an additional three equations of the same type. Solving the system which consists of these additional three equations together with eq. (1.21) for the coefficients $A_{n}, B_{n}, C_{n}$ and $D_{n}$, and then substituting their values in (1.20), we get

$$
Q^{*}\left(|n|, k_{1}, k_{2}\right) G_{n-}=-P_{n-}^{*}+G_{n+}
$$

where

$$
\begin{aligned}
Q^{*}\left(|n|, k_{1}, k_{2}\right) & =\frac{\left\{2 \mu n^{2}-(\lambda+2 \mu) k_{1}^{2}\right\}\left(k_{2}^{2}-2 n^{2}\right)}{k_{2}^{2} \sqrt{n^{2}-k_{1}^{2}}} \operatorname{coth} \sqrt{n^{2}-k_{1}^{2}}+\frac{4 \mu n^{2} \sqrt{n^{2}-k_{2}^{2}}}{k_{2}^{2}} \operatorname{coth} \sqrt{n^{2}-k_{2}^{2}} \\
& =2 \mu \frac{k_{2}^{2}-k_{1}^{2}}{k_{2}^{2}}|n|+O\left(|n|^{-1}\right)
\end{aligned}
$$

Finally, the discrete problem (1.22) can be written in the standard form

$$
|n| G_{n-}+Q_{|n|} G_{n_{-}}=-P_{n_{-}}+G_{n_{+}}^{*}
$$

where

$$
\begin{aligned}
& Q_{|n|}=\frac{k_{2}^{2}}{2 \mu\left(k_{2}^{2}-k_{1}^{2}\right)} Q^{*}\left(|n|, k_{1}, k_{2}\right)-|n| \\
& P_{n-}=\frac{k_{2}^{2}}{2 \mu\left(k_{2}^{2}-k_{1}^{2}\right)} P_{n-}^{*} \text { and } G_{n+}^{*}=\frac{k_{2}^{2}}{2 \mu\left(k_{2}^{2}-k_{1}^{2}\right)} G_{n+}
\end{aligned}
$$

\section{REDUCTION OF THE DISCRETE PROBLEM TO AN ALGEBRAIC SYSTEM OF EQUATIONS}

Applying the inverse Fourier transform to eq. (1.24), we get

$$
\frac{1}{\pi i} \frac{d}{d x} \int_{-\pi}^{\pi} \frac{G_{-}(x) d t}{1-e^{i(x-t)}}+\sum_{n=-\infty}^{\infty} Q_{|n|} G_{n} e^{i n x}=-P+P_{+}^{*}(x)
$$

where

$$
P=\frac{k_{2}^{2}}{2 \mu\left(k_{2}^{2}-k_{1}^{2}\right)} P^{*}, \quad G_{+}^{*}(x)=\frac{k_{2}^{2}}{2 \mu\left(k_{2}^{2}-k_{1}^{2}\right)} G_{+}(x) .
$$

Recalling that $G_{+}^{*}(x)=0$ for $-c<x<c$ and integrating eq. (2.1) with respect to $\theta$, we obtain over the interval $-c<x<c$

$$
\frac{1}{\pi i} \int_{-c}^{c} \frac{G_{-}(x) d t}{1-e^{i(x-t)}}+Q_{0} G_{0-} \sum_{n-\infty}^{\infty} \frac{(-1)^{n}}{n i} e^{i n x}+\sum_{n-\infty}^{\infty} Q_{|n|} G_{n} \frac{e^{i n x}}{i n}=-P \sum_{n=-\infty}^{\infty} \frac{(-1)^{n}}{n i} e^{i n x}+\alpha
$$

where $\alpha$ is constant and the primes over the summation symbols mean that the value $\boldsymbol{n}=\mathbf{0}$ is not included. Here we have used the expansion

$$
x=\sum_{n=-\infty}^{\infty} \frac{(-1)^{n}}{n i} e^{i n x} .
$$

The singular integral equation with the Cauchy kernel (2.3) possesses a bounded solution $G_{-}(x)$ which can be written in the form (Eckhardt and El-Sheikh [2])

$$
G_{-}(x)=-R(x)\left[Q_{0} G_{0}-\sum_{n--\infty}^{\infty} \frac{(-1)^{n}}{n} I_{n}(x)+\sum_{n-\infty}^{\infty} Q_{|n|} \frac{G_{n-}}{n} I_{n}(x)+P \sum_{n=-\infty}^{\infty} \frac{(-1)^{n}}{n} I_{n}(x)-i \alpha I_{0}(x)\right]
$$

where 


$$
\begin{gathered}
I_{n}(x)=\frac{1}{\pi} \int_{-c}^{c} \frac{e^{i(n+1) \xi} d \xi}{R(\xi)\left(e^{i \xi}-e^{i x}\right)}, \\
R(x)=\lim _{\substack{z\left|e^{a x}\\
\right| z \mid<1}} \sqrt{\left(z-e^{i c}\right)\left(z-e^{-i c}\right)} .
\end{gathered}
$$

The integrals $I_{n}(x)$ are to be understood in the sense of the principal value. For $n>0,[3$, p. 215], we have

$$
I_{n}(x)=-e^{i(n-1) x} \sum_{j=0}^{n-1} \frac{e^{-i x j}}{2^{j}} \sum_{m=0}^{j} \frac{(2 m-1) ! ![2(j-m)-1] ! !}{m !(j-m) ! e^{i m c} e^{-i(j-m) c}}, \quad n>0
$$

and

$$
I_{0}(x)=0 \text {. }
$$

Further, it has already been shown (Muskhelishvili [4]) that

$$
I_{-n}(x)=-e^{-i x} I_{n}(-x) \text {. }
$$

In relation (2.8), the convention $0 ! !=(-1) ! !=1$ is adopted. By virtue of $(2.9)$ and the fact that $\frac{G_{n-}}{n}$ is odd, the solution (2.5) can be written in the form

$$
\begin{aligned}
G_{-}(x)= & -R(x)\left[Q_{0} G_{0}-\sum_{n=1}^{\infty} \frac{(-1)^{n}}{n}\left\{I_{n}(x)-I_{-n}(x)\right\}\right. \\
& \left.+\sum_{n=1}^{\infty} Q_{n} \frac{G_{n}-}{n}\left\{I_{n}(x)-I_{-n}(x)\right\}+P \sum_{n=1}^{\infty} \frac{(-1)^{n}}{n}\left\{I_{n}(x)-I_{-n}(x)\right\}\right] .
\end{aligned}
$$

Substituting in the formula

$$
G_{l-}=\frac{1}{2 \pi} \int_{-c}^{c} G_{-}(x) e^{i l x} d x
$$

according to (2.11), we get the algebraic system

$$
G_{t-}+Q_{0} G_{0-} N_{\alpha_{l}}+\sum_{n=1}^{\infty} Q_{n} \frac{G_{n-}}{n}\left[N_{n l}-N_{-n l}\right]=P N_{0 l}, \quad l=0,1,2, \ldots
$$

where for $n \neq 0$ we have used the notation

$$
\begin{aligned}
N_{-n l} & =\frac{1}{2 i} \int_{-c}^{c} R(x) I_{-n}(x) e^{-i l x} d x \\
& =\sum_{j=0}^{n-1} A_{j-n-l-1} \sum_{m=0}^{j} \frac{(2 m-1) ! ![2(j-m)-1] ! !}{2 m ! !(2 j-2 m) ! ! e^{i(j-2 m) c}} \\
N_{n l} & =-\sum_{j=0}^{n-1} A_{n-j-l-2} \sum_{m=0}^{j} \frac{(2 m-1) ! ![2(j-m)-1] ! !}{2 m ! !(2 j-2 m) ! ! e^{-i(j-2 m) c}} .
\end{aligned}
$$

where

$$
A_{k}=\frac{1}{2 \pi i} \int_{X B} \sqrt{(t-A)(t-B)} t^{k} d t, \quad A=e^{-i c}, \quad B=e^{i c}
$$

and the $\operatorname{arc} A B$ is directed along the unit circle. The values of these integrals are given by the formulas $[3$, p. 216] 


$$
\begin{aligned}
A_{-1}= & \frac{e^{-t c}+e^{\imath c}}{4}-\frac{1}{2}=A_{-2}, \\
A_{-k}= & \frac{1}{2^{k}}\left[\frac{(2 k-5) ! !\left[e^{-i(k-1) c}+e^{i(k-1) c}\right]}{(k-1) !}\right. \\
& \left.-\sum_{m=1}^{k-2} \frac{(2 m-3) ! !(2 k-2 m-5) ! !}{m !(k-1-m) ! e^{i(k-1-2 m) c}}\right], \quad k>2 .
\end{aligned}
$$

and for $k>0$ we have [4]

$$
A_{k}=A_{-(k+3)} .
$$

Finally, $N_{0}$ is given by

$$
N_{0 l}=\sum_{n=1}^{\infty} \frac{(-1)^{n}}{n}\left\{N_{n l}-N_{-n l}\right\}
$$

It has been shown [2] that $N_{-n}\left(N_{l n}\right)$ tends to zero as $l \rightarrow \infty$ more rapidly than $\frac{1}{l}$ and indeed, it tends to zero as $n \rightarrow \infty$ since

$$
\frac{1}{l} N_{-l n}\left(N_{l n}\right)=\frac{1}{n} N_{-n l}\left(N_{n l}\right)
$$

This establishes not only that the coefficients of $G_{n_{-}}, n=0,1,2, \ldots$ in system (2.13) will tend to zero as $n, l \rightarrow \infty$, but also that the expression of $N_{\alpha}$ will converge so quickly that it can be calculated through truncation at a suitable order.

\section{JUSTIFICATION OF THE TRUNCATION}

The infinite system (2.13) can be solved by truncating it at a suitable order $m$. This is equivalent to reducing (2.3) to the more simple equation

$$
\tilde{K} \tilde{G}_{-}=\tilde{f}
$$

where $\tilde{K}$ and $\tilde{G}$ are "approximations" to the operator $K$ and the function $G_{-}(x)$, respectively, in the left-hand side of the original equation (2.3)

$$
\tilde{K} \tilde{G}=\frac{1}{\pi i} \int_{-c}^{c} \frac{\tilde{G}_{-}(x) d t}{1-e^{i(x-t)}}+Q_{0} \tilde{G}_{0}-\sum_{n=-\infty}^{\infty} \frac{(-1)^{n}}{n i} e^{i n x}+\sum_{n=-m}^{m} Q_{|m|} \tilde{G}_{n}-\frac{e^{i n x}}{i n}
$$

and

$$
\tilde{f}=-P \sum_{n=-m}^{m} \frac{(-1)^{n}}{n i} e^{i n x}+\tilde{\alpha}
$$

Equation (2.13) itself can be denoted similarly in the form

$$
K G_{-}=f \text {. }
$$

We shall now outline some features about equations (3.1) and (3.4). First, eq. (3.1) has a unique bounded solution defined by eq. (2.5) truncated at the m-th order and in which $\left\{G_{n_{-}}, n=0,1, \ldots, m\right\}$ is the solution of system (2.13) truncated at the m-th order. Second, the Banach space $L_{2}[-c, c]$ is the domain and range of both the operators $K$ and $\tilde{K}$. Finally, the operator $\tilde{K}^{-1}(K-\tilde{K})$ is bounded in $L_{2}[-c, c]$ with the norm

$$
\left\|\tilde{K}^{-1}(K-\tilde{K})\right\|<1,
$$

provided $m$ is sufficiently large. This will be established if we show that $\|K-\tilde{K}\|$ can be made arbitrarily small. We have 


$$
(K-\tilde{K}) G(x)=\frac{1}{\pi} \sum_{n=m}^{\infty} \frac{Q_{n}}{n} \int_{-c}^{c} \sin n(x-t) G(t) d t
$$

and consequently,

$$
\begin{aligned}
\|(K-\tilde{K}) G(x)\| & =\int_{-}^{c}\left|\frac{1}{\pi} \sum_{n=m}^{\infty} \frac{Q_{n}}{n} \int_{-c}^{c} \sin n(x-t) G(t) d t\right|^{2} d x \\
& \leq \frac{1}{\pi^{2}} \int_{-c}^{c}\left(\sum_{n=m}^{\infty} \frac{Q_{n}}{n} \int_{-c}^{c}|G(t)| \cdot|\sin n(x-t)| d t\right)^{2} d x \\
& \leq \frac{1}{\pi^{2}}\left(\sum_{n=m}^{\infty} \frac{Q_{n}}{n} \int_{-c}^{c}|G(t)| d t\right)^{2} \int_{-c}^{c} d x \\
& =\frac{2 c}{\pi^{2}}\left(\sum_{n=m}^{\infty} \frac{Q_{n}}{n} \int_{-c}^{c}|G(t)| d t\right)^{2} \\
& =\frac{2 c}{\pi^{2}}\left(\sum_{n=m}^{\infty} \frac{Q_{n}}{n}\right)^{2}\left(\int_{c}^{c}|G(t)| d t\right)^{2} \\
& \leq \frac{2 c}{\pi^{2}}\left(\sum_{n=m}^{\infty} \frac{Q_{n}}{n}\right)^{c} d t \int_{-c}^{c}|G(t)|^{2} d t \\
& \leq 4\left(\sum_{n=m}^{\infty} \frac{Q_{n}}{n}\right)^{2}\|G\|^{2}
\end{aligned}
$$

from which it follows that

$$
\|K-\tilde{K}\| \leq 2 \sum_{n-m}^{\infty} \frac{Q_{n}}{n} .
$$

The right-hand side in the last expression tends to zero since $Q_{n}=0\left(\frac{1}{n}\right)$ according to the definitions (1.25) and (1.23), and the relation (3.5) will eventually be fulfilled.

In view of the above considerations (Cherski [5]), it follows that equation (2.13) has the unique solution

$$
G_{-}=\tilde{G}_{-}+\left[I+\tilde{K}^{-1}(K-\tilde{K})\right]^{-1} \tilde{K}^{-1}(f-K \tilde{G}),
$$

where $I$ is the unit operator. Further, the resulting error due to the truncation can be estimated according to the formula

$$
\left\|G_{-}-\tilde{G}_{-}\right\| \leq \frac{\left\|\tilde{K}^{-1}\left(f-K \tilde{G}_{-}\right)\right\|}{1-\left\|\tilde{K}^{-1}(K-\tilde{K})\right\|}
$$

\section{REFERENCES}

[1] NOWACKI, W., Thermoelasticity, Pergamon Press, PWN, Oxford-Warsava, 1962.

[2] ECKHARDT, U., EL-SHEIKH, M. G., "A Fourier Method for Solving Initial Boundary Value Problems with Mixed Boundary Conditions," Computers and Mathematics with Applications, Vol. 14, No. 3, pp. 189-199, 1987.

[3] GAKHOV, F. D., CHERSKI, Ju. I., "The Equations of Convolution Type," Nauka, Moscow, 1978 (Russian).

[4] MUSKHELISHVILI, N. I., Singular Integral Equations, Noordhoff, Groningen, 1953.

[5] CAERSKI, JU, I., "Two Theorems on Estimation of the Error and Some of Their Applications, " Dikl. Akad. Nauk SSSR, 150 (1963).

*Present Address: Mathematics Department, Faculty of Science, King Abdulaziz University, P.O. Box 9028 Jeddah, Saudi Arabia. 


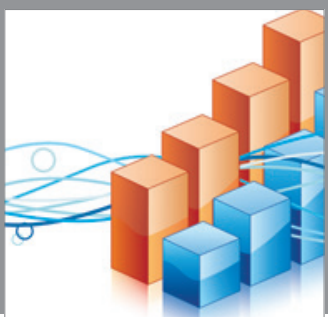

Advances in

Operations Research

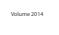

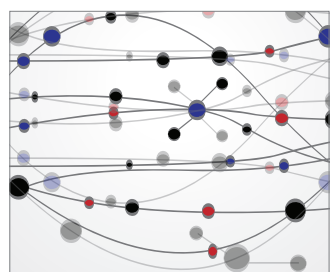

\section{The Scientific} World Journal
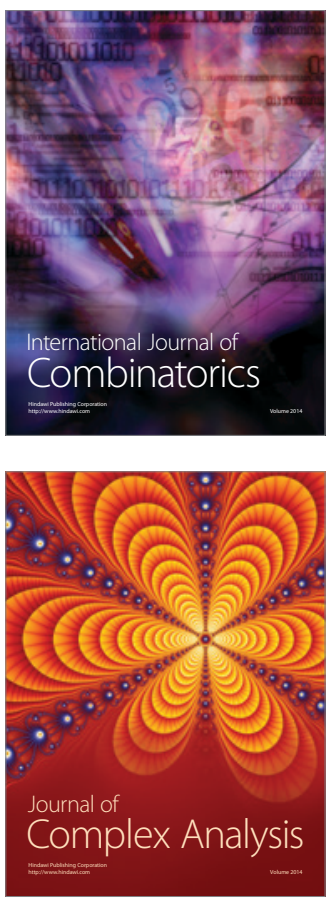

International Journal of

Mathematics and

Mathematical

Sciences
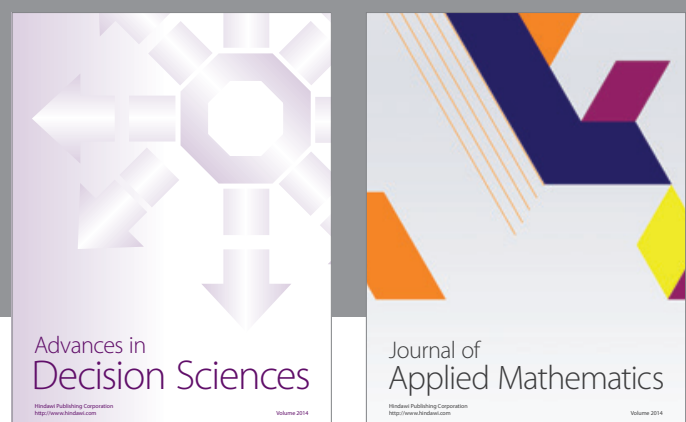

Journal of

Applied Mathematics
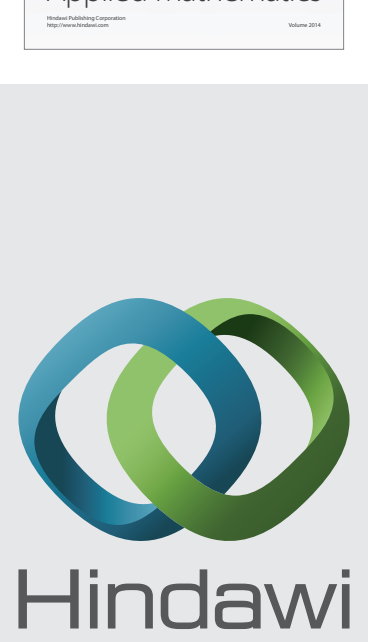

Submit your manuscripts at http://www.hindawi.com
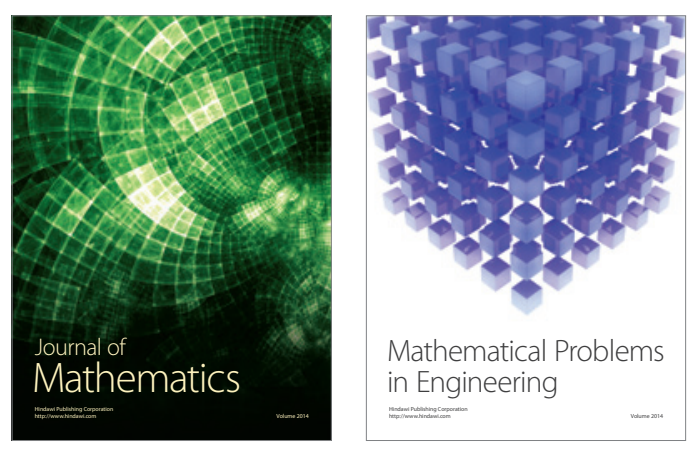

Mathematical Problems in Engineering
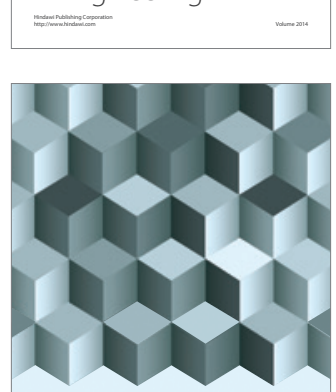

Journal of

Function Spaces
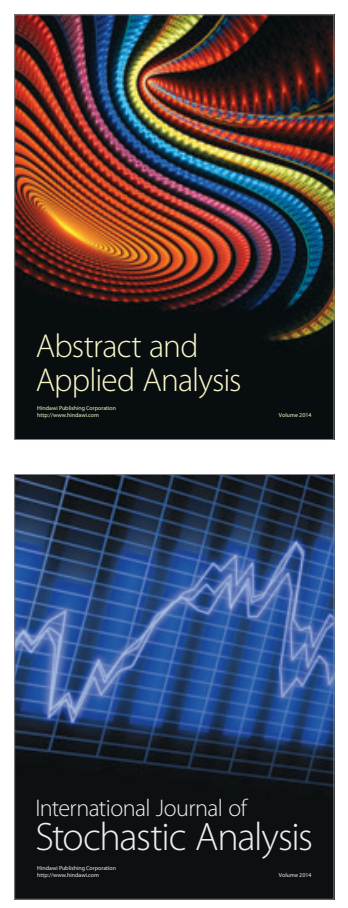

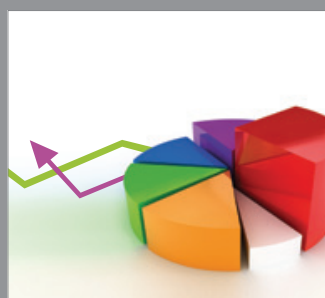

ournal of

Probability and Statistics

Promensencen
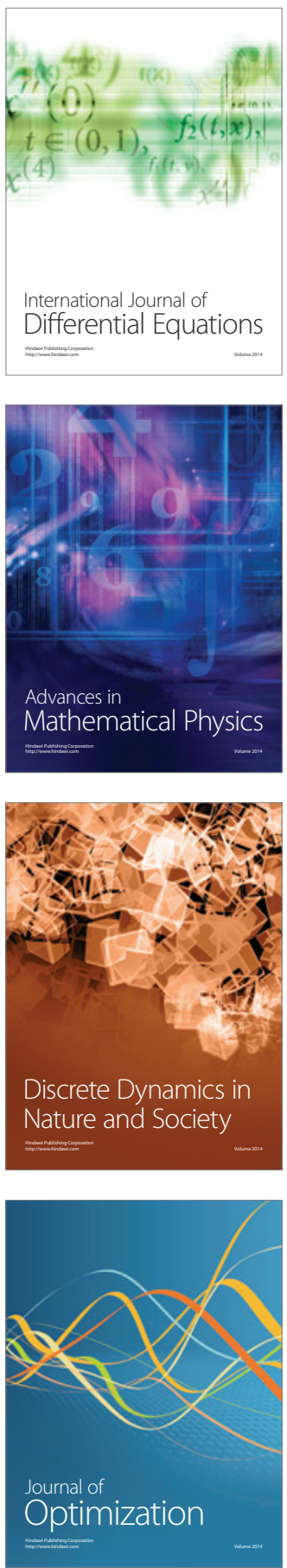